\title{
Evaluation on Service Ability of Agri-Food Supply Chain
}

\author{
Liu Qiao ${ }^{1, *}$, Zhao Dawei ${ }^{1}$ and Shen Xin ${ }^{2}$ \\ ${ }^{I}$ Management School, Harbin University of Science and Technology, Harbin, Heilongjiang, 150080 China; \\ ${ }^{2}$ Management School, Harbin University of Commerce, Harbin, Heilongjiang, 150028, China
}

\begin{abstract}
This study aims to develop a comprehensive framework to evaluate the service ability of agri-food supply chain based on Chinese food industry. We develop an evaluation index system with five aspects resepectively, transportation, wareshousing \& storage, customer response, distribution and information level. With analytic hierarchy process to identify the weight of each index, we apply fuzzy comprehensive evaluation method to evaluate the overall system. A numberic example shows that the evaluating system can be applied not only for agricultural enterprises to select cold chain service suppliers, but also for these suppliers to analyze their own service ability to identify the oppertunities for improvement.
\end{abstract}

Keywords: Agri-food, fuzzy comprehensive evaluation, service ability, supply chain.

\section{INTRODUCTION}

Currently, the agri-food supply chain has received a great deal of attention [1]. Due to deficient supply chain service ability [2], there are approximately over 12 million tons of fruits and 11.3 billion tons of vegetables deteriorated before reaching to the end users in China. The 10-billion losses could meet the produce demands for millions of people. This concern has been strengthened by several sector-wide crises $[3,4]$. How to eliminate the waste in the process and improve the efficiency of supply chain is a big challenge to Chinese food industry as well as academia. Hence we aim to establish an applicable evaluation framework to assess the agri-food supply chain service ability based on Chinese practice so as to push the improvement of Chinese logistics enterprises and benefit the Chinese food industry.

It is essential to define the agri-food supply chain service ability before discussion. From the perspective of value chain, the formation of core service ability, to great degree, relies on the all inputs and capital arrangement in the activities of logistics and supply chain operation $[5,6]$. For the logistics and supply chain operators, different input modes of the factors and capital differentiate the service ability of operators, which, in some sense, is the source of competitive advantages for the enterprises as little total costs as possible. The customer service ability and organization operational ability, as this argument goes, are the main valuable principles to the respective drive of demand and supply. As far as the agri-food supply chain, the service ability of whole supply chain will also be of great significance to the produce quality safety [7]. In this study, we define the agri-food supply chain service ability (ASCSA) as the comprehensive implication for all the applicable supply chain methods mix applied by the agri-food supply chain operators to keep the perishable agri-food in the appropriate temperature from points of origin to points of consumption, which may be concern with produce elementary processing, storage, transportation, distribution and sales, for the purpose to assure agri-food quality, eliminate deterioration and meet the demands of customers. This service ability may be represented as response level, logistics cost, order fill rate and delivery reliability and so on.

Before we establish the evaluation framework of ASCSA, we also necessitate detailed analysis on the composition of ASCSA. With the constraint of enterprise resources, different service suppliers differ in service operation [8-9]. According to the service object and relevant evaluation, we describe ASCSA as internal ASCSA and external ASCSA. Internal ASCSA refers as the inner system operational ability to support supply chain, which serve inner members of the chain and coordinate the efficient and effective upstream and downstream operation. This is generally evaluated by the inner members of the chain. As for the external ASCSA, it is the externalized power maintaining the supply chain and entities outside of the chain, which works on the interactivity of incomings and outgoings. Thus external ASCSA can be directly perceived or evaluated by the external entities. Standing merely at the evaluable perspective, ASCSA is composed of service level, hardware level and software level [10]. Service level concludes reliability, customer satisfaction, post-transaction service and service flexibility [11]. Hardware level refers transportation, warehousing and delivery ability. Software level is concerned with information technology including RFID application to produce tracing and positioning etc, as well as organizational strategy [12]. As can be seen, few literatures shed light on the service ability of agri-food supply chain, and we try to illustrate the issue based on Chinese practice. 
Table 1. The ASCSA framework.

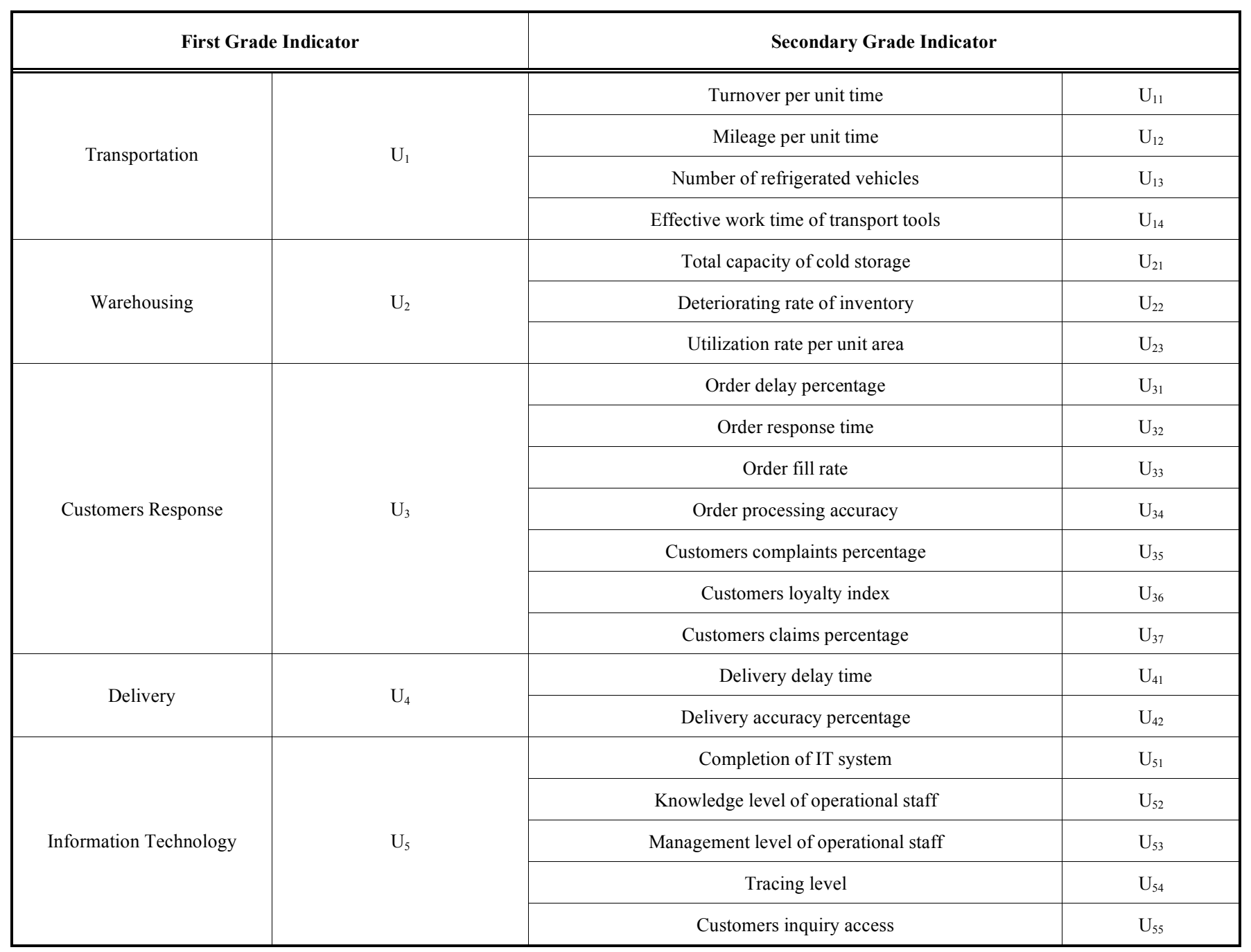

2. ESTABLISHMENT OF EVALUATION INDEX SYSTEM

This study aims to analyze the ASCSA based on Chinese practice. Before evaluating, it is an initial stage for the study to shed light on a framework with relevant evaluating indicators.

\subsection{Identification of Evaluation Framework}

On the basis of above discussion about agri-food supply chain and service ability, we identified first grade assessment indicators, respectively, Transportation Capability, Warehousing Capability, Customers Response, Delivery Capability and Information Technology Level. Subsequently, we identified second grade indicators accordingly. These indicators were determined through reference review and qualitative analysis with Nvivo. Also, a questionnaire was generated and counterbalanced before distributing to experts in academia and industry. As result, an evaluation index framework is shown as Table $\mathbf{1}$.

In the first grade indictors, $U_{1}$ includes four subindicators. $\mathrm{U}_{11}$ refers total produce turnover realized by the supply chain operators in specific time bond. $U_{12}$ refers the higher indicator, the more efficient transportation. Due to the perishability of produce, more number of refrigerated vehicles means the more chance to eliminate deterioration in transit, which is represented by $U_{13}$. In China, the percentage of such logistics vehicles is rather low as Table 2. The figures in Table 2 are calculated with Chinese 680 logistics enterprise whose avenue in 2014 above 10 million RMB. Considering plenty of small enterprises, we estimate total refrigerated vehicles would be roughly 50000 , which occupied around $0.3 \%$ of commercial trucks, much lower than the percentage in developed countries, like US (1\%) and Germany $(3 \%)$. The cold trucks per 10 thousand individuals in 2014 were up to 0.36 units, and that of US were 8 units. $U_{14}$ means the effective working time of transport tools in specific time limit without maintenance, repairing, parking and other time in which the facilities fail to utilize.

$\mathrm{U}_{21}$ is another important factor in produce logistics competitive. The statistics also presents total capacity of cold storage in above enterprises as Table 3. The figures were $20,469,713$ tons, equivalent as $57,546,093$ cubic meters. The capacity of cold storage per person in 2014 is up to 0.015 tons, twice higher than that of 2010, but much lower than that of US (0.2 tons). $\mathrm{U}_{22}$ represents the ratio of inventory 
Table 2. Refrigerated vehicles numbers in Chinese provinces and Municipalities in 2014.

\begin{tabular}{|c|c|c|c|c|c|}
\hline Ranking & Province/Municipality & $\begin{array}{c}\text { Refrigerated } \\
\text { Vehicles\# }\end{array}$ & Ranking & $\begin{array}{c}\text { Province/ } \\
\text { Municipality }\end{array}$ & Refrigerated Vehicles\# \\
\hline 1 & Shanghai & 8736 & 16 & Hubei & 310 \\
\hline 2 & Henan & 5031 & 17 & Hunan & 304 \\
\hline 3 & Beijing & 3915 & 18 & Sichuan & 195 \\
\hline 4 & Chongqing & 1378 & 19 & Shanxi & 187 \\
\hline 5 & Shandong & 1194 & 20 & Zhejiang & 183 \\
\hline 6 & Fujian & 1164 & 21 & Shaanxi & 181 \\
\hline 7 & Anhui & 1129 & 22 & Gansu & 140 \\
\hline 8 & Guangdong & 984 & 23 & Jiangxi & 109 \\
\hline 9 & Neimenggu & 782 & 24 & Hainan & 90 \\
\hline 10 & Tianjin & 752 & 25 & Jilin & 72 \\
\hline 11 & Jiangsu & 621 & 26 & Guizhou & 45 \\
\hline 12 & Heilongjiang & 605 & 27 & Xinjiang & 43 \\
\hline 13 & Niaoning & 563 & 28 & Guangxi & 13 \\
\hline 14 & Hebei & 387 & 29 & Ningxia & 4 \\
\hline 15 & Yunnan & 327 & \multicolumn{2}{|c|}{ Total \# } & 29444 \\
\hline
\end{tabular}

Table 3. Total capacity of cold storage in Chinese provinces and Municipalities in 2014.

\begin{tabular}{|c|c|c|c|c|c|}
\hline 1 & Jiangsu & $3,204,274$ & 16 & Shaanxi & 291,832 \\
\hline 3 & Shandong & $1,917,048$ & 18 & Jilin & 267,567 \\
\hline 4 & Hunan & $1,647,126$ & 19 & Hubei & 250,210 \\
\hline 6 & Henan & 982,702 & 21 & Heilongjiang & 225,000 \\
\hline 7 & Beijing & 961,449 & 22 & Shanxi & 199,548 \\
\hline 8 & Chongqing & 823,533 & 23 & Guangxi & 193,892 \\
\hline 12 & Fujian & 557,666 & 27 & Gansu & 161,872 \\
\hline 13 & Neimenggu & 535,189 & 28 & Ningxia & 139,601 \\
\hline 14 & Hebei & 427,638 & 29 & Guizhou & 97,333 \\
\hline 15 & Xinjiang & 426,166 & \multicolumn{2}{|c|}{ Total Capacity } & $20,469,713$ tons \\
\hline
\end{tabular}

deteriorated because of lack of cold chain facilities. $U_{23}$ is the good indicator to evaluate the warehousing efficiency.

$\mathrm{U}_{31}$ is the ratio of order delayed to fulfill according to the designated time with the customers. $\mathrm{U}_{32}$ represents how promptly the enterprises response against the information passed on from customers. $\mathrm{U}_{33}$ is used to another efficient KPI. $U_{34}$ reflects the processing accuracy. $U_{35}, U_{36}$ and $U_{37}$ reflect the customers' direct perception to supply chain serv- 
Table 4. ASCSA Review for the sample company.

\begin{tabular}{|c|c|c|c|c|c|c|c|}
\hline \multirow{2}{*}{ Criterion } & \multirow{2}{*}{ Weight } & \multicolumn{5}{|c|}{ Membership Degree } \\
\cline { 3 - 8 } & & Excellent & Very good & Good & Fair & Poor & Score \\
\hline \hline Transportation & 0.32 & 0.152 & 0.259 & 0.294 & 0.117 & 0.252 & 74.310 \\
\hline Warehousing & 0.32 & 0.092 & 0.192 & 0.255 & 0.211 & 0.336 & 68.415 \\
\hline Customers Response & 0.15 & 0.299 & 0.252 & 0.185 & 0.248 & 0.163 & 84.430 \\
\hline Delivery & 0.15 & 0.117 & 0.250 & 0.383 & 0.183 & 0.125 & 74.825 \\
\hline InformationTechnology & 0.06 & 0.204 & 0.298 & 0.203 & 0.162 & 0.054 & 71.010 \\
\hline Total & & & 73.83 & & \\
\hline
\end{tabular}

ices. Once a customer satisfies with the service of one supply chain operator, makes repeated purchasing of services, the preference would form to some loyalty, which is usually influenced by many factors like service quality, price, convenience etc. In china, low loyalty would be a challenge to enterprises, not only in logistics and supply chain. There are a lot of companies driven on price, which result in plenty of price-sensitive customers. Agri-food chain is not a valueintensive chain, and customers are always tolerant to the bad -quality-but-low-price service. With the economic development, this phenomenon would be decreasing but not to be ignored in Chinese market. The dramatic fresh produce consuming demand with the huge lack of cold chain means that customers' claims would also be a lasting problem which enterprises pretend to neglect.

$\mathrm{U}_{41}$ and $\mathrm{U}_{42}$ represent the delivery delay and accuracy. Information technology $\left(\mathrm{U}_{5}\right)$ is a huge gap between China and developed countries in supply chain, which includes the condition of IT system like MIS in these forms, knowledge and management level of personnel, and some specific factors like tracing and customs inquiring system (Gui, L., Z.W. He. etc, 2010).

\subsection{Determination of Weight}

Remarks set as follows,

$$
V=\left(\begin{array}{llll}
V_{1} & V_{2} & V_{3} & \ldots . . V_{m}
\end{array}\right)
$$

In the study, we determine $m$ as 5 , so the remark for ASCSA V $=\{$ excellent, very good, good, fair, poor $\}$. We apply AHP method to assign the weight of every indicator. Initially, after the above hierarchy model established, we make a pairwise comparison of the importance of a certain criteria in same hierarchy to its upper hierarchy, and construct two comparative judgment matrix so as to analyze the weight.

Weight is as follows,

$$
\omega=\left(\begin{array}{lll}
\omega_{1} & \omega_{2} & \omega_{3} \ldots . . . \omega_{m}
\end{array}\right)
$$

where $\omega_{i}$ is the weight of second grade indicator. Total comparison result represents as

$$
\mathrm{A}=\left(\mathrm{A}_{\mathrm{ij}}\right)_{\mathrm{m} \times \mathrm{n}}
$$

where $m^{\prime}$ and $n^{\prime}$ are the number of second and third evaluation indicators; $A$ is judgement matrix; $A_{i j}$ values as scale 19 according to reference 3 . After that, we need to check consistency to the judgement matrix as follows:

(1) Calculate the consistency of A

$$
C I=\lambda_{\max }-\mathrm{n} \phi(n-1)
$$

where $\mathrm{n}$ is the order of judgement matrix and $\lambda_{\max }$ is the maximum eigenvalue of $\mathrm{A}$.

$\ominus$ Inquiry average random consistency indicator RI.

$\circledast$ Calculate the consistency percentage

$$
C R=C I / R I
$$

when $C R<0.1$, we accept the consistency of the judgment or a pairwise comparison would be repeated and adjust the matrix to have the acceptable consistency.

\section{(4) Identify the evaluation membership matrix.}

The relationship of each evaluation factor and its evaluating grade, the Fuzzy relation from $\mathrm{U}$ to $\mathrm{V}$, can be described with evaluation membership matrix as follows,

$$
\mathrm{R}=\left(\begin{array}{cccc}
R_{11} & R_{12} & \cdots & R_{15} \\
R_{21} & R_{22} & \cdots & R_{25} \\
\cdots & \cdots & \cdots & \cdots \\
R_{P 5} & R_{P 2} & \cdots & R_{P 5}
\end{array}\right)
$$

where $\mathrm{p}$ is the number of next level indicator included by upper indicator system, and $\mathrm{p}=2,3,4,5,7$

The membership degree of the second and third indicator to evaluation $\mathrm{M}$ grade is represented as $\gamma_{p m}$,

$$
0 \leq \gamma_{p m} \leq 1
$$

\subsection{Fuzzy Comprehensive Evaluation}

The expression of weight $\omega_{\text {and evaluation membership }}$ matrix $\mathrm{R}$ is

$$
\mathrm{A} \circ R=\left(\begin{array}{lllll}
b_{1} & b_{2} & b_{3} & b_{4} & b_{5}
\end{array}\right)=\mathrm{B}
$$


where the grade membership degree with indicator to remark as b1,b2,.....b5; fuzzy arithmetic operators is " $\circ$ ".

Through the first fuzzy comprehensive evaluation, the membership degree $\mathrm{B}$ of the first grade indictor to evaluation grade can be counted. Subsequently we apply B as the fuzzy relationship matrix $Q$ as the second fuzzy comprehensive evaluation to get the second fuzzy comprehensive result for ASCSA, where

$Q=\left(\begin{array}{llll}\mathrm{B}_{1} & \mathrm{~B}_{2} & \cdots & \mathrm{B}_{n}\end{array}\right)^{T}, \mathrm{~B}=\mathrm{A} \circ Q$

Lastly, quantifying the factors of remark set and calculate the results with expression

$P_{\mathrm{i}}=\mathrm{B}_{\mathrm{i}} V^{T}$

\section{NUMBERICAL EXAMPLE}

\subsection{Identification of Weight}

A agri-food supply chain company in Heilongjiang province, China operates mainly produce cold (cool) storage, transportation and distribution. It has six large cold vehicles with temperature recording in whole process. The main operating area of the company covers most cities in Heilongjiang, Jilin, Liaoning, Neimenggu, Shandong and Anhui, with door to door service and supply chain scheduling for the customers. Also the company can supply services such as cargo tracking, Cash on delivery, FCL (full carload) and LCL(less carload). We invited ten experts to give scores to the indicator of this company according to specialist marking methods. After constructing and normalizing of judgement matrix, the weight of second level to first level indicator as follows,

$$
\omega=\left(\begin{array}{lllll}
0.32 & 0.32 & 0.15 & 0.15 & 0.06
\end{array}\right)
$$

From above we can identify that the crucial factors impacting to its ASCSA are, namely, transport, warehousing, customers response and distribution ability. We applied square root method to calculate the mix eigenvalue of $\mathrm{A}$ is 5 , $\mathrm{S}$ is 5 , and $\mathrm{RI}$ is 1.12 .

According to expression formula (4) and (5), we can find $\mathrm{CI}=0, \mathrm{CR}=0$, so the consistency of judgement matrix A can be accepted.

\subsection{Fuzzy Comprehensive Evaluation}

We invited those experts to score for the membership degree of the second indicator remark sets, and took the average score as the membership degree of some indicator to remark set. Similarly, we obtained the membership degree of third factors set to remark set. Accordingly, every factor evaluation affiliation matrix R1 $\sim \mathrm{R} 5$ can be calculated.

With the formula (8), the first fuzzy evaluation result comes as follows:

$$
\begin{aligned}
\mathrm{B}_{1} & =\mathrm{A}_{1} \circ R_{1}=\left[\begin{array}{lllll}
0.152 & 0.259 & 0.294 & 0.117 & 0.252
\end{array}\right] \\
\mathrm{B}_{2}= & {\left[\begin{array}{lllll}
0.092 & 0.192 & 0.255 & 0.211 & 0.336
\end{array}\right] } \\
\mathrm{B}_{3} & =\left[\begin{array}{lllll}
0.229 & 0.252 & 0.185 & 0.248 & 0.163
\end{array}\right] \\
\mathrm{B}_{4} & =\left[\begin{array}{lllll}
0.117 & 0.250 & 0.383 & 0.183 & 0.125
\end{array}\right] \\
\mathrm{B}_{5} & =\left[\begin{array}{lllll}
0.204 & 0.298 & 0.203 & 0.162 & 0.054
\end{array}\right]
\end{aligned}
$$

After obtaining the first level to remark set membership degree, second fuzzy comprehensive evaluation carried out with the results of $\mathrm{B}$ as follows:

$$
\mathrm{B}=\left[\begin{array}{lllll}
0.160 & 0.240 & 0.267 & 0.154 & 0.227
\end{array}\right]
$$

As can be seen, the maximum membership degree is 0.267 . Accordingto the principle of maximum membership degree, the general review is quite satisfied and we can understand the ASCSA of that company is medium.

\subsection{Review Analysis}

Hereby the qualified value of each factor in the Set V is as follows

$$
\mathrm{V} 1=100, \mathrm{~V} 2=85, \mathrm{~V} 3=70, \mathrm{~V} 4=55, \mathrm{~V} 5=40
$$

Clearly, the closer to 100, the higher ASCSA of the company; the closer to 40 , the poorer ASCSA. We use the expression to calculate the score as follows

$\mathrm{P}_{i}=\mathrm{B}_{i} V^{T}$

Table $\mathbf{4}$ is the result of above calculation. From the comprehensive score, we can analyze the supply chain service ability of the company. It is easy to find the company has rather high customers services ability but has great space to improve its transport and warehousing ability. Generally speaking, the ASCSA of the company is between good and very good.

Based on the practice of the sample company, we identified the main reasons of the lack of ASCSA in following aspects. At the outset, the turnover in unit time was rather low. We suggested the company to improve its schedule service level. Although the company has the sufficient number of rather advanced cold chain facilities, there is still far way to improve the service quality and response speed with the increasing demand of customers. Therefore the second suggestion we gave to the company is to introduce relevant cold chain facilities like vehicles according to the category of cargoes. Also, we found the precooling technology is not enough applied in the service process, which to some degree, resulted in deterioration of produce. Besides these urgent aspects for the company to improve, another reason lowering the service ability is due to low utilization per square meters of cold warehouse. Admittedly, it results from complicated factors. In order to lower the deteriorating rate of produce, it is essential for all supply chain operators to give enough attachment to the scientific warehousing planning and layout. In addition, despite the medium service ability in delivery and information level, the upgrading some software and hardware is quite needed to meet the increasing demand of consumers and coping with the intensive market competition.

\section{CONCLUSION}

This study aim to evaluate the agri-food supply chain service ability for those operating enterprises based on AHP and fuzzy comprehensive evaluation methods. The evaluating system for this purpose is based on Chinese data, which can be applied not only for agricultural enterprises to select cold chain service suppliers, but also for these suppliers to assess their own service ability so as to improve their competitiveness in the market. However, due to the high diver- 
sity of agriculture products like the giant difference in characteristics of fruits and vegetables, meats and fishes, it is difficult to apply one single evaluation framework to all sorts of segmentation of cold chain market. Taking weather as example, North China considerably differs in the natural condition of South China, the big temperature distance for above areas would be as large as approximately 60 centigrade. Also customers' expectation from different regions is different; in other words, the tolerance of customers to service in developed region is generally not as sensitive as that in developing areas like West China. All these problems would be challenges for operators as well as the further topic for Chinese academia to study.

\section{CONFLICT OF INTEREST}

The authors confirm that this article content has no conflict of interest.

\section{ACKNOWLEDGEMENTS}

This work is supported by follows: Humanities and Social Science Research Project of Ministry of Education, P.R. China (No.13YJA630139), and China Scholarship Council (No. 201308230120).

\section{REFERENCES}

[1] O. Ahumada, and J.R. Villalobos, "Application of planning models in the agri-food supply chain: A review," European Journal of Operational Research, vol. 4, pp. 1-20, 2009.
[2] K. Balachandran, and R. Suresh, "Quality Implications of Warranties in a Supply Chain," Management Science, vol. 51, pp. 1266$1277,2005$.

[3] J. Blandon, S. Henson, and T. Islam, "The Importance of Assessing Marketing Preferences of Small-scale Farmers: A Latent Segment Approach," European Journal of Development Research, vol. 22, pp. 494-509, 2010.

[4] L. Gui, Z.W. He, and L.J. Zhao, "Construction of Agri-food Logistics Information Platform in Beijing," Wuhan: Hubei Peoples Press, pp. 1015-1018, 2013. (in Chinese)

[5] J. G. A. J. van der Vorst, "Product traceability in food-supply chains," Accreditation and Quality Assurance, vol. 11, pp. 3337, 2006.

[6] K.H. Lai, T.C.E. Cheng, and A.C.L. Yeung, "Relationship Stability and Supplier Commitment to Quality," International Journal of Production Economics, vol. 3, pp. 397-400, 2005.

[7] H. Huo, X. Shen, and Z. Huang, "Analysis of Supply Chain Medel of Agricultural Products and Quality Safety," Asian Agricultural Research, vol. 10, pp. 50-53, 2011

[8] C. J. Robinson, and M. K. Malhot, "Two-commodity reliability evaluation for a stochastic-flow network with node failure," Computers and Operations Research, vol. 13, pp. 1927 1939, 2002.

[9] N. Seth, S.G. Deshmukh, and P. Vrat, "A framework for measurement of quality of service in supply chains," Supply Chain Management, vol. 11, pp. 82-94, 2006.

[10] J.L. Wang, "A supply chain application of fuzzy set theory to inventory control models - DRP system analysis," Expert Systems with Applications, vol. 36, pp. 9229-9239, 2009.

[11] S. Xin, "Application of Vector Markov Chain to Reliability Evaluation of Agricultural Products Cold Chain," Advances in Information Sciences and Service Science, vol. 4. no. 19, pp. 554-560, 2012.

[12] B. Yan, S. Shi, and B. Ye, "Sustainable development of the fresh agricultural products supply chain through the application of RFID technology," Information Technology \& Management, vol. 16. pp. 67-78, 2015.

(C) Qiao et al.; Licensee Bentham Open.

This is an open access article licensed under the terms of the (https://creativecommons.org/licenses/by/4.0/legalcode), which permits unrestricted, noncommercial use, distribution and reproduction in any medium, provided the work is properly cited. 